\section{Ohne Ziele keine Führung Situativer Führungsstil heißt, seine Mitarbeiter „mitzunehmen“}

\begin{abstract}
Seit 2004 ist Andreas Schlüter, diplomierter Verwaltungswirt, Geschäftsführer des Klinikums Westfalen GmbH. Das Klinikum in Trägerschaft der Knappschaft Bahn See verfügt seit den Fusionen 2010 und 2013 über 3 Krankenhausstandorte mit insgesamt 818 Betten und 1800 Beschäftigten in Dortmund, Kamen und Lünen. In den Häusern werden jährlich 35000 Patienten stationär und knapp 100000 Patienten ambulant behandelt. Der Umsatz liegt bei 130 Millionen Euro. Seit April 2013 managt Schlüter zudem die Geschicke der Klinikum Vest GmbH, einem Klinikum in ähnlicher Größenordnung, mit einem Knappschaftshaus in Recklinghausen und der Paracelsus Klinik in Marl. Zusammen erzielen die beiden Kliniken einen Umsatz von knapp 300 Millionen Euro pro Jahr. Die Mitarbeiter gelten als ihr wichtigstes Kapital. Das derzeit größte Problem ist die Arbeitsverdichtung auf den Stationen.
\end{abstract}

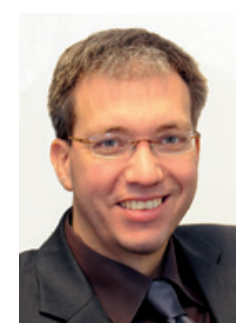

Andreas Schlüter

\section{? Wird in den Häusern des Klinikums Vest anders geführt als im Westfalen Klinikum?}

Andreas Schlüter: Für den gesamten Verbund der Knappschaft und damaligen Knappschaftskliniken haben wir ein einheitliches Leitbild entwickelt, das der Vorstand Ende der 90er Jahre genehmigt hat. Unsere Führungskräfte sollen Orientierung geben und die Eigenverantwortung ihrer Mitarbeiter stärken. Natürlich gibt es auch Unterschiede in und zwischen den Kliniken. Die Häuser bringen ihre eigene Kultur mit, die den formalen Zusammen- schluss überdauert. Zum Beispiel organisiert der Ärztliche Direktor die Chefarztsitzungen im Klinikum Westfalen, ich nehme in der Regel an jeder zweiten Sitzung teil. Das Klinikum Vest fordert traditionell die Anwesenheit der Geschäftsführung stärker ein. Eine Chefarztsitzung kann schon mal ausfallen, wenn ich nicht da bin.

Schlüter: Wir führen nach Zielen, das war bei den städtischen Häusern vor der Fusionierung so nicht immer der Fall. Unsere

\section{? Welche Strategien entwickeln Sie, um trotz Arbeitsverdichtung die Motivation Ihrer Mitarbeiter zu erhalten?}

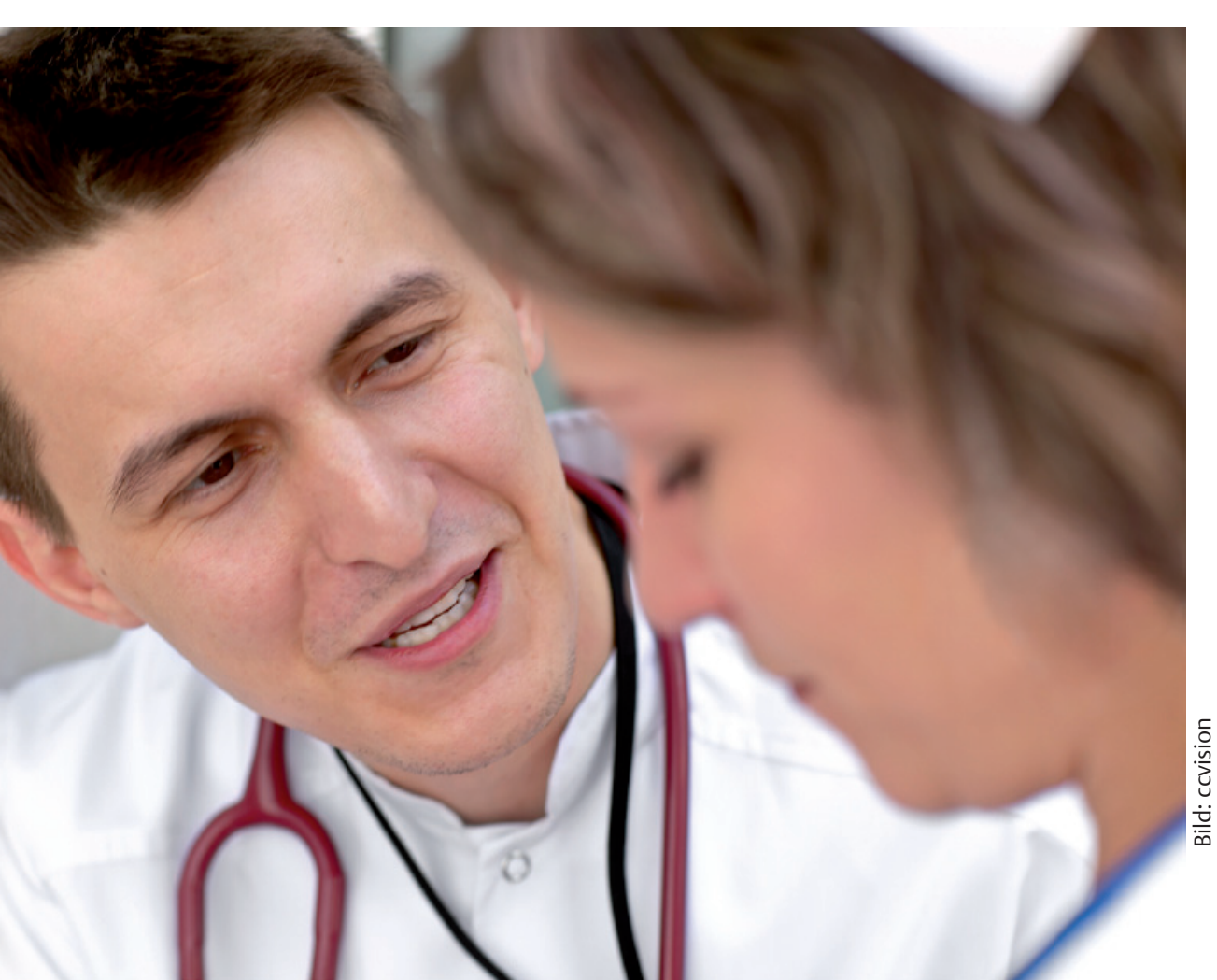

Zielvorgaben machen wir transparent. Sie sollen allen Mitarbeitern bekannt sein, nicht nur der obersten Leitungsebene. Ebenso informieren wir die Mitarbeiter über die Ergebnisse, die erreicht worden sind, einschließlich der wirtschaftlichen Daten. Die Arbeit im Krankenhaus wollen wir verlässlich machen. Mittlerweile erfassen auch Ärzte und Pflegekräfte ihren Zeitaufwand auf den Stationen und bei Projekten, sodass Überstunden abgebaut oder entgolten werden können. Gerade die junge Generation legt Wert auf Struktur und Planbarkeit. Deswegen wollen wir Ärzte bereits zu Dienstbeginn informieren, wenn Überstunden anfallen und nicht erst kurz vor Dienstschluss. Um unser Ausbildungsversprechen einzuhalten, bauen wir eine Akademie auf und garantieren, dass angehende Fachärzte die nötigen Untersuchungszahlen tatsächlich in der vorgegebenen Zeit realisieren können. In der Anästhesie können wir dies bereits messen. Des Weiteren haben wir mit dem Betriebsrat eine leistungsorientierte Prämie für Mitarbeiter eingeführt. Zusätzlich stellen wir pro Mitarbeiter ein freies Bildungsbudget in Höhe von 500 Euro zur Verfügung, über dessen Verwendung die Abteilungen entscheiden.

? Ist ein demokratischer Führungsstil im Krankenhaus möglich, der den Teams in der Verwaltung und auf den Stationen eine hohe Entscheidungsautonomie zugesteht?

Schlüter: Wir setzen auf einen situativen Führungsstil, bei dem wir unsere Mitarbeiter in Entscheidungs- und Entwicklungsprozesse der Häuser einbeziehen, wann immer es möglich ist. Ein hohes Ausmaß an demokratischen Entscheidungsprozessen ist in Projekten gegeben. Zum Beispiel mussten im Klinikum Vest die Standorte der Pneumologie und der Gefäßchirurgie getauscht werden, um für beide Bereiche die jeweils besten Bedingungen zu erreichen. Der gesamte Prozess der Neu- und Umstrukturierung ist in Arbeitsgruppen vorbereitet worden. Die dort getroffenen Entscheidungen konnten wir nahezu vollständig umsetzen. Beim Standortwechsel sind alle Mitarbeiter mitgegangen. In der Patientenversorgung setzt ein demokratischer Stil voraus, dass sich jeder gleich verantwortlich fühlt. Ein Beispiel sind Tumorkonferenzen, wo Fachärzte unterschiedlicher Disziplinen auf gleicher Ebene miteinander über den bestmöglichen Behand- 
lungsweg diskutieren und zu einer Entscheidung kommen müssen. Auch in der Bereichspflege, wo auf einer Station 4 Pflegekräfte 35 bis 40 Patienten betreuen, ist ein hohes Maß an Selbstorganisation vorhanden. Autokratisch und chefarztzentriert verlaufen Entscheidungsstrukturen hingegen, wenn Wissen, Kompetenzen und Verantwortung unterschiedlich verteilt sind.

\section{? Was erwarten Sie von Chefärzten und anderen Führungskräften?}

Schlüter: Führungskräfte der Knappschaftshäuser müssen teamfähig sein und ihre Mitarbeiter motivieren können. Sie müssen Zielvorgaben weiterentwickeln und für ihre Abteilung oder Station konkretisieren. Wenn Verlässlichkeit ein hoher Wert ist, muss sich dies in der Terminplanung niederschlagen. Wenn das Traumazentrum ausgebaut werden soll, müssen Kapazitäten geplant werden, sodass auch in Notfällen ein freies Bett und qualifiziertes Personal zur Verfügung stehen. Chefärzte und Stationsleitungen müssen ihre Mitarbeiter „mitnehmen“. Das ist möglich, wenn sie ihre Ziele nach der SMART-Formel spezifisch, messbar, transparent, akzeptabel, realistisch, terminiert und vor allem transparent ma- chen, Feedback geben und Mitarbeitergespräche führen.

\section{? Wo sehen Sie Handlungsbedarf?}

Schlüter: Teamfähigkeit ist nicht immer gegeben. Chefärzte und Assistenzärzte repräsentieren unterschiedliche Generationen. Ärzte, die am Anfang ihrer beruflichen Karriere stehen, müssen lernen, sich in bestehende Strukturen zu integrieren, während viele ältere Chefärzte ihren dominanten, autokratischen Führungsstil hinterfragen müssen. Wir bieten hierzu Coachings an. Wenn Teamfähigkeit bei Chefärzten fehlt, ist dies ein Grund zur Trennung. Für Führungskräfte, die von der zweiten in die erste Führungsebene wechseln, mieten wir Schulungszentren, um die Gruppe gezielt auf ihre neuen Führungsaufgaben vorzubereiten. Um eine standortübergreifende Teamfähigkeit zu schaffen, ermöglichen wir Mitarbeitern zeitweise an einem anderen Standort zu arbeiten. Wir arbeiten an unserem Ziel, dass sich die Mitarbeiter mit dem jeweiligen Klinikum identifizieren, statt mit dem Standort der ehemals eigenständigen Häuser. Bei den Mitarbeitern der Vestischen Kliniken ist letzteres noch verbreitet. Wir sind dabei, die unterschiedlichen Kulturen der Häuser zu- sammenzuführen und Synergien zu fördern, beispielsweise durch das Zusammenlegen von Funktionsbereichen. Die Arbeit an den Schnittstellen ist ein fortwährender Prozess.

Bergsteiger Reinhold Messner sprach auf dem Gesundheitskongress des Westens davon, dass derjenige Leadership hat, der sich am stärksten mit dem Unternehmen identifiziert. Ist das auf ein Krankenhaus übertragbar? Und auf Sie persönlich?

Schlüiter: Beides kann ich bejahen. Die Arbeit im Krankenhaus erfordert eine $100 \%$ ige Identifikation mit den Unternehmenszielen. Gerade für Ärzte in verantwortungsvoller Position ist der Beruf ein Teil ihres Lebens. Sie müssen bereit sein, in Notfällen Privates zurückzustellen, um für den Patienten da zu sein. Mit einer "Jobmentalität“ wäre das nicht möglich. Am Ende zählt das Ergebnis. Wer sich mit den Zielen identifiziert, arbeitet auch hart daran, sie zu erreichen.

\section{Herr Schlüter, vielen Dank für das} Gespräch!

Das Interview führte Dr. Adelheid Weßling, Düsseldorf 